\title{
An Overview of Raster Scanning for ICF-Class Laser Optics
}

\author{
M. Runkel, M. C. Nostrand
}

This article was submitted to Boulder Damage Symposium XXXIV: Annual Symposium on Optical Materials for High Power Lasers, Boulder, CO, September 16-18, 2002

\section{October 30, 2002}

U.S. Department of Energy

Lawrence

Livermore

National

Laboratory

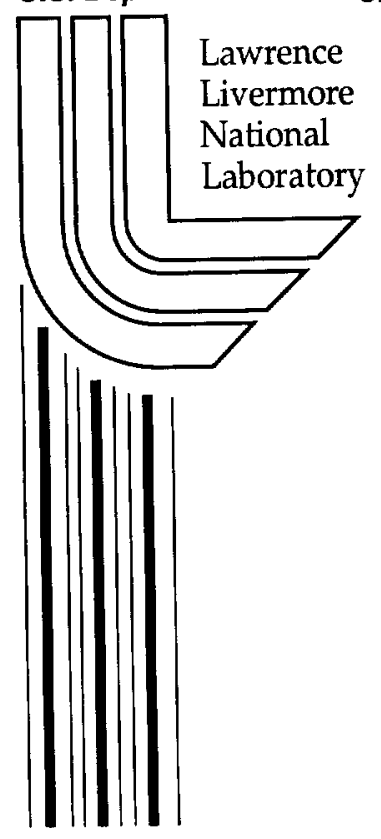




\section{DISCLAIMER}

This document was prepared as an account of work sponsored by an agency of the United States Government. Neither the United States Government nor the University of California nor any of their employees, makes any warranty, express or implied, or assumes any legal liability or responsibility for the accuracy, completeness, or usefulness of any information, apparatus, product, or process disclosed, or represents that its use would not infringe privately owned rights. Reference herein to any specific commercial product, process, or service by trade name, trademark, manufacturer, or otherwise, does not necessarily constitute or imply its endorsement, recommendation, or favoring by the United States Government or the University of California. The views and opinions of authors expressed herein do not necessarily state or reflect those of the United States Government or the University of California, and shall not be used for advertising or product endorsement purposes.

This is a preprint of a paper intended for publication in a journal or proceedings. Since changes may be made before publication, this preprint is made available with the understanding that it will not be cited or reproduced without the permission of the author.

This work was performed under the auspices of the United States Department of Energy by the University of California, Lawrence Livermore National Laboratory under contract No. W-7405-Eng-48.

This report has been reproduced directly from the best available copy.

Available electronically at http://www.doc.gov/bridge

Available for a processing fee to U.S. Department of Energy

And its contractors in paper from

U.S. Department of Energy

Office of Scientific and Technical Information

P.O. Box 62

Oak Ridge, TN 37831-0062

Telephone: (865) 576-8401

Facsimile: (865) 576-5728

E-mail: reports@adonis.osti.gov

Available for the sale to the public from

U.S. Department of Commerce

National Technical Information Service 5285 Port Royal Road

Springfield, VA 22161

Telephone: (800) 553-6847

Facsimile: (703) 605-6900

E-mail: orders@ntis.fedworld.gov

Online ordering: http://www.ntis.gov/ordering.htm

\section{OR}

Lawrence Livermore National Laboratory

Technical Information Department's Digital Library

http://www.llnl.gov/tid/Library.html 


\title{
An overview of raster scanning for ICF-class laser optics
}

\author{
Michael Runkel and Mike C. Nostrand \\ Lawrence Livermore National Laboratory \\ P.O. Box 808, L-491 Livermore, CA 94550 \\ Phone: (925)-424-2210, runkel1@1lnl.gov
}

\begin{abstract}
Recent work has shown that the damage resistance of both ICF-class $\left(1600 \mathrm{~cm}^{2}\right)$ DKDP tripler crystals and $\mathrm{SiO}_{2}$ components (lenses, gratings and debris shields) benefits from laser raster scanning using pulsed lasers in the $350 \mathrm{~nm}$ range. For laser raster scanning to be a viable optical improvement tool for these large optics, damage improvement must be optimized while maintaining scan times of less than 8 hours/optic. In this paper we examine raster scanning with small beams from tabletop laser systems. We show that 120 Watts of average power is required for a tabletop scanning system at one optic/day. Next, we develop equations for total scan time for square and round top hat beams and round and rectangular gaussian beams. We also consider the effect of packing geometry (square vs. hexagonal), examine the deviations from uniform coverage with each scan geometry and show that hexagonal packing yields lower scan times but is less efficient in coverage than square geometry. We also show that multiple passes at low packing densities are temporally equivalent to a single pass with higher packing density, and discuss the advantages of each method. In addition, we show that the differences between hexagonal and square scan geometries are negated when pointing errors and fluence fluctuations from the laser are considered.
\end{abstract}

\section{INTRODUCTION}

Past experience has shown that the damage resistance of virtually all optical components found in Inertial Confinement Fusion (ICF) lasers benefits from low fluence laser exposures (conditioning) at their use wavelength.

Conditioning is a generic term that means different things for different optical materials. For a coated optic at $1053 \mathrm{~nm}$ $(1 \omega)$ it is the benign removal of nodular coating defects. ${ }^{1}$ For $\mathrm{SiO}_{2}$ and DKDP at $355 \mathrm{~nm}(3 \omega)$ the process is less clear. In the case of $\mathrm{SiO}_{2}$, conditioning could be as simple as benign removal of trapped residual polishing compounds (i.e. laser cleaning), or as complicated as changing the surface structure. ${ }^{2}$ Similarly, for DKDP conditioning could be the reduction in the size or optical absorption of an absorbing defect, or the reduction in the density of a currently unknown type of defect structure. ${ }^{3}$ Even though the details of the conditioning process in DKDP are unknown, it is still possible to take advantage of the effect.

In principle, conditioning can and will be achieved on-line during beam activation ${ }^{4}$, but there are many concerns associated with it, the major one being its spatial uniformity. This stems from the fluence distribution of the beam for a single pulse, as well as the repeatability of the spatial profile on a shot-to-shot basis. In addition, on-line conditioning can put many components at risk. We have seen in testing of a prototype final optic assembly (FOA) on LLNL's Beamlet laser that damage on one component caused contamination and damage on others. ${ }^{5}$ A third reason is the cost of firing the laser. Although on-line conditioning will occur as part of the initial beamline activation, this type of conditioning is undesirable when optics will be replaced as part of normal maintenance, and this will occur in the middle of experimental campaigns. Clearly the best scenario for both beamline activation and normal optic replacement cycles is to provide the laser with maximally conditioned components from an off-line source. By this we mean that the component has been exposed to a laser in such a way as to uniformly decrease its damage defect concentration to a minimum (practical) value over the clear aperture. This places some constraints on the types of systems that can be used for conditioning, especially when treating approximately 1000 ICF-class optics. The ideal system for conditioning would be a single ICF beamline with true top-hat spatial profile and high pulse repetition frequency. But, because of issues mentioned above, this is not practical. For similar reasons neither are smaller "large beam" systems like LLNL's Optical Sciences Laser. In addition, the smaller beam requires stitching together of multiple shots to fill the optic's clear aperture. By extending this to its logical conclusion, one arrives at the notion of scanning an optic with a high rep-rate source using a small beam. One could then have control of the laser and scan parameters to fill the optic with needed 
beam fluence and desired uniformity. Such a system can be constructed from commercially available, millijoule class, "table-top" lasers.

This idea has been around for several years. To date, both laser glass slabs and turning mirrors have been scanned by systems at $1 \omega$ [references 3 and 6] while KDP/DKDP crystals and fused silica components will be scanned at $3 \omega$. For the $1 \omega$ optics, the laser glass slabs are scanned using top-hat beam profiles while the mirrors are scanned using gaussian spatial profiles. Until now, $3 \omega$-optics have only been scanned using gaussian beams, because top-hat beam delivery systems are substantially more complicated. In the following sections we examine the factors involved in gaussian-beam based raster-scanning systems at $3 \omega$.

\section{AVERAGE POWER REQUIREMENTS}

The final optics assembly for the latest generation of ICF class lasers will nominally consist of harmonic generation crystals (KDP and DKDP), focus lens, beam separation grating and debris shield $\left(\mathrm{SiO}_{2}\right)$. Current estimates based on initial build rate and subsequent refurbishment of optics place the maximum optical throughput at up to eight FOA units per week. It is straightforward to determine the laser power requirements needed to meet this level of throughput. The scan time per conditioning pass is given by

$$
t=\frac{A}{a R}=\frac{A F}{E R}=\frac{A F}{P}
$$

Here, $A$ is the area to be scanned, a is the beam area, $\mathrm{R}$ is the laser repetition rate, $\mathrm{E}$ is the pulse energy, $\mathrm{F}$ is the beam fluence and $\mathrm{P}$ is the average laser power. As a baseline we assume uniform coverage by a square top hat beam with maximum scan fluence of $14 \mathrm{~J} / \mathrm{cm}^{2}$ at $3 \mathrm{~ns}$. The power required to complete $1600 \mathrm{~cm}^{2}$ in 8 hours is 0.78 Watts. For a 10-pass conditioning protocol $\left(4-14 \mathrm{~J} / \mathrm{cm}^{2}\right)$ the power requirement is 7.8 Watts. Considering optical losses of $50 \%$ and a $30 \%$ safety factor the required power is 20 Watts. To achieve the desired $8 \mathrm{FOA} /$ week would require $120 \mathrm{~W}$ of laser power provided a square top hat profile at $3 \mathrm{~ns}$ is available.

\section{THE EFFECT OF BEAM SHAPE ON SCAN TIME}

The four beam shapes that are being considered for raster scanning are square top hat (STH), round top hat (RTH), round gaussian (RG) and rectangular gaussian (gaussian short axis, top hat long axis, 1D). In scanning applications where the leading edge of the beam is not needed for conditioning ${ }^{7}$, the square top hat is clearly the optimum choice since it offers uniform coverage with no overlap of successive pulses. The round top hat is next, but requires small overlap for uniform coverage. The two gaussian beams are less desirable because a significant portion of the beam energy is below the peak fluence, effectively lowering the usable area of the beam. However, because gaussian beams are more readily produced by tabletop lasers they merit consideration and are the central focus of this paper. We look at each case below.

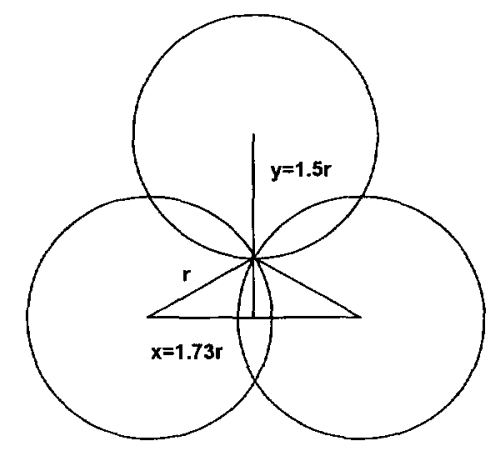

Figure 1. Geometry for hexagonal packed round top hat beams. The cell formed by horizontal and vertical steps $\mathrm{x}$ and $\mathrm{y}$ is $21 \%$ smaller than for the beam itself, increasing scan time by $21 \%$. 


\subsection{Scan time for round top hat}

Figure 1 shows the geometry of hexagonal packing of round top hat beams for uniform coverage. In order to achieve this packing the horizontal step size must be $1.73 * \mathrm{r}$ or $0.866 * \mathrm{~d}$ where $\mathrm{d}$ is the beam diameter. The vertical step size is $0.75 * \mathrm{~d}$. The area of this cell is $21 \%$ smaller than for the round top hat so the scan will take $21 \%$ longer.

\subsection{Scan time for gaussian beams}

In raster scanning the goal is to uniformly irradiate a large region with a small laser beam. Typically the sample is moved at constant speed in one direction. At the end of the scan line, the sample is shifted orthogonal to the scan direction and the scan direction is reversed. The scan speed depends on the beam size ( $\phi$, laser repetition rate $(\mathrm{R})$ and the fill factor $(\mathrm{N}=$ number of shots per beam diameter $)$ and can be calculated according to $\mathrm{v}=\phi \mathrm{R} / \mathrm{N}$. The total scan time can be calculated according to $\mathrm{T}=\mathrm{A} /(\mathrm{v} \Delta)$ where $\mathrm{A}$ is the total scan area and $\Delta$ is the shift between scan lines. When using beams with gaussian components (see Figure 2) it is apparent that the scan region cannot be uniformly irradiated and there will be fluence "ripple" that depends on the fill factor.

$$
\begin{aligned}
& \begin{array}{l}
\text { Rectangular } \\
\text { gaussian }
\end{array} \\
& A_{e f f}=\frac{\sqrt{\pi}}{2 \sqrt{2}} l \phi \\
& \begin{array}{l}
\mathrm{I}=\mathrm{FWHM}, \text { long axis } \\
\phi=1 / \mathrm{e}^{2} \text { diameter, } \\
\text { short axis }
\end{array}
\end{aligned}
$$
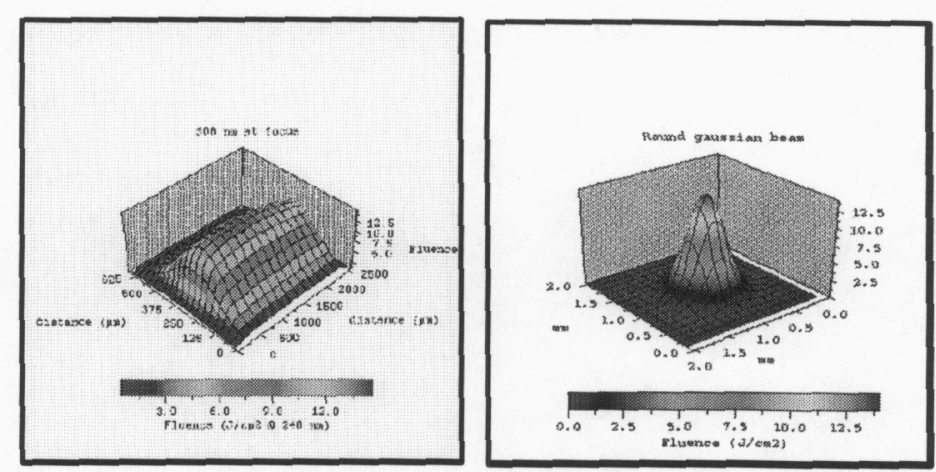

Round gaussian

$$
\begin{gathered}
A_{\text {eff }}=\frac{\pi \phi^{2}}{8} \\
\phi=1 / \mathrm{e}^{2} \text { diameter }
\end{gathered}
$$

Figure 2. Beams with gaussian components. The left plot shows a rectangular gaussian with short axis width $\left(1 / \mathrm{e}^{2}\right)$ of $\phi$ and long axis width (FWHM) of 1 . The right plot shows a round gaussian beam of width $\phi$

Figure 3 shows a 1-dimensional view of the overlap of gaussian laser beams.

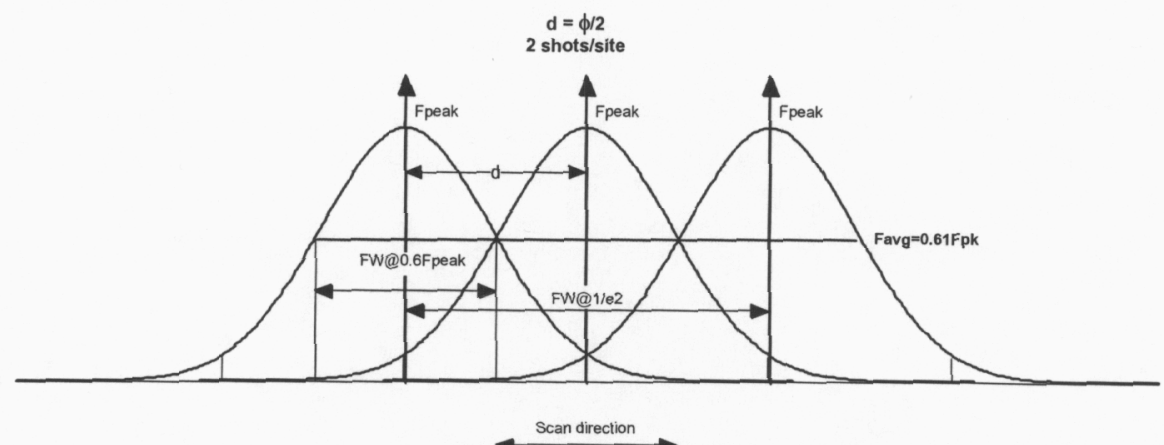

Figure 3. Gaussian spatial profile with width $\phi$ and $d$ corresponding to $F_{N=2}(r)=0.61 F_{p k}$. This corresponds to a fill factor of 2 shots/site.

Using the $F(r)=F_{p k} \exp \left[-8\left(r^{2} / \phi^{2}\right)\right]$ and $N=\phi / d$, we find the relation between the beam ripple and fill factor. 


$$
N=\frac{1}{\sqrt{0.5 \ln \left(F_{p k} / F_{N}\right)}}=\frac{\sqrt{2}}{\sqrt{\ln (1 / f)}}
$$

Here $\phi$ is the $1 / \mathrm{e}^{2}$ beam diameter and $\mathrm{d}$ represents the diameter at fluence F. We call $f$ the beam overlap. For the onedimensional case the ripple factor is simply $1 / f$ but for two-dimensional round gaussians it depends on whether the packing geometry is square or hexagonal. From geometric considerations it can be shown that the lowest fluence in the scan region occurs at the center of a scan "cell". We call this ratio of this fluence to $\mathrm{F}_{p k}$ the minimum fill fraction (MFF). The square and hexagonal packing geometries are shown in Figure 4.

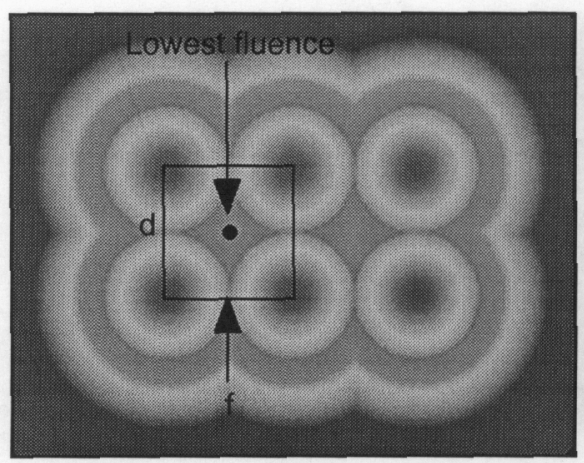

Cubic packing

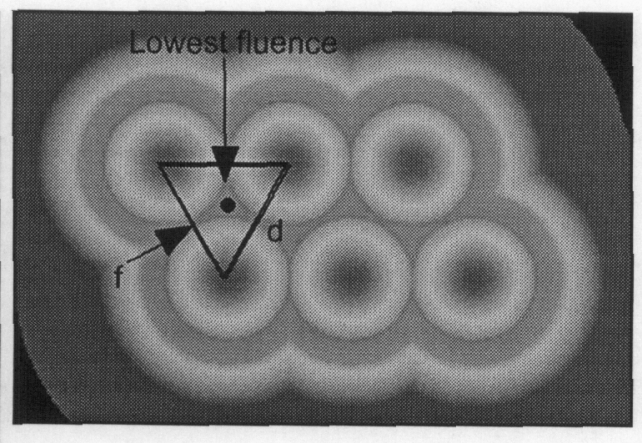

Hexagonal packing

Figure 4. Square and hexagonal packing for shots in raster scanning. The low fluence point in the scan occurs at the geometric center of the cell. The point corresponding to the scan overlap, $f$, is also indicated in the figures.

The MFF values for these packing geometries are given in term of the fill factor $\mathrm{N}$ by

$$
M F F_{1 D}=\exp \left(-2 / N^{2}\right), M F F_{\text {square }}=\exp \left(-4 / N^{2}\right), M F F_{\text {hex }}=\exp \left(-8 / 3 N^{2}\right)
$$

The total scan time equation becomes $\mathrm{T}_{\text {square }}=\mathrm{AN}^{2} / \mathrm{R} \phi^{2}$ for square packing, while for hex packing it is $\mathrm{T}_{\text {hex }}=$ $2 \mathrm{AN}^{2} / 3^{0.5} \mathrm{R} \phi^{2}$. Thus for the same $\mathrm{N}$, the hexagonal scan time is $15.5 \%$ longer (since the vertical shift $\mathrm{D}$ is $15.5 \%$ smaller.) Conversely, for the same values of MFF we have $T_{\text {hex }} / T_{\text {cubic }}=4 / 3 \sqrt{3}$ indicating that the hexagonal packing takes only $77 \%$ of the square packing time. Inversion of equations (3) and insertion into the scan time equation $\mathrm{T}=\mathrm{A} /(\mathrm{v} \Delta)$ allows the scan time to be written in terms of the MFF.

$$
T_{1 D}=\frac{A \sqrt{2}}{\phi R l \sqrt{1 / M F F}}, T_{\text {square }}=\frac{4 A}{R \phi^{2} \ln (1 / M F F)}, T_{\text {hex }}=\frac{16 A}{3 \sqrt{3} R \phi^{2} \ln (1 / M F F)}
$$

Rewriting these equations in terms of laser power and peak fluence (equation 1) allows scan times to be compared for equivalent MFF values. The scan times for these beams depends on the MFF and are longer than for the square top hat (STH). This is shown in Figure 5.

$$
\frac{T_{1 D}}{T_{S T H}}=\frac{\sqrt{\pi}}{2 \sqrt{\ln (1 / M F F)}}, \frac{T_{\text {square }}}{T_{S T H}}=\frac{\pi}{2 \ln (1 / M F F)}, \frac{T_{\text {hex }}}{T_{S T H}}=\frac{2 \pi}{3 \sqrt{3} \ln (1 / M F F)}
$$




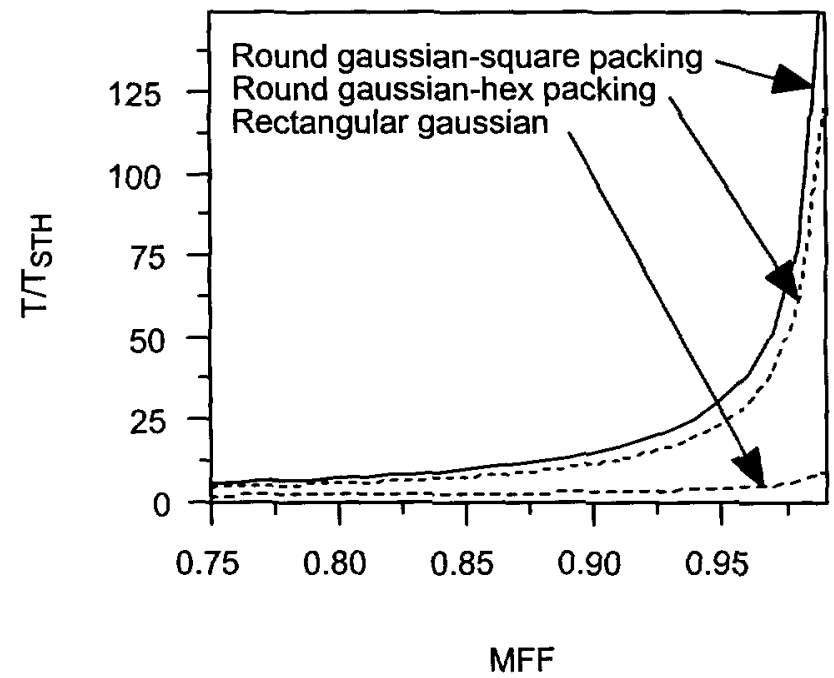

Figure 5. Relative scan times as a function of MFF for round gaussian beams with square and hexagonal packing and rectangular (1D) gaussian beams based on equations (5). For equivalent average power and fluence the rectangular gaussian beam gives the lowest scan times for any MFF and becomes substantially faster at MMF values above 0.8 .

\subsection{Effectiveness of packing geometry for round gaussian beams}

From the standpoint of using the MFF as a figure of merit, it may appear that the hexagonal packing scheme is more desirable, but this is not necessarily true. In addition to the MFF, we need to know what fraction of the scanned area $\left(\mathrm{A}_{\mathrm{eff}} / \mathrm{A}\right)$ has been exposed to what fraction of the peak fluence $\left(\mathrm{F} / \mathrm{F}_{\mathrm{pk}}\right)$. This fractional area can be determined on a geometrical basis by calculating the area of the circle of radius corresponding to the (arbitrary) gaussian fluence $\mathrm{F}$, relative to the area of the square (square case) or hexagonal unit cell, see Figure 6. Such a plot is shown in Figure 7. For completeness, the formulas are given by

$$
\begin{aligned}
\frac{\mathrm{A}_{\mathrm{eff}}}{\mathrm{A}}=\frac{\pi}{2} \varepsilon, & 0 \leq \varepsilon \leq 1 / 2 & \text { (cubic) } \\
=\frac{\pi}{2} \varepsilon+\sqrt{2 \varepsilon-1} & -2 \varepsilon \cos ^{-1}[\sqrt{1 /(2 \varepsilon)}], & 1 / 2 \leq \varepsilon \leq 1
\end{aligned}
$$

and

$$
\begin{array}{rrr}
\frac{\mathrm{A}_{\mathrm{eff}}}{\mathrm{A}}=\frac{2 \pi}{3 \sqrt{3}} \varepsilon, & 0 \leq \varepsilon \leq 3 / 4 & \text { (hexagonal) } \\
=\frac{2 \pi}{3 \sqrt{3}} \varepsilon+\sqrt{4 \varepsilon-3} & -\frac{4}{\sqrt{3}} \varepsilon \cos ^{-1}[\sqrt{3 /(4 \varepsilon)}], & 3 / 4 \leq \varepsilon \leq 1
\end{array}
$$

where $\varepsilon=\ln \left(\mathrm{F}_{\mathrm{pk}} / \mathrm{F}\right) / \ln (1 / \mathrm{MFF})$. 
d

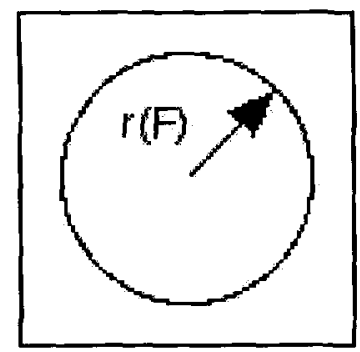

$\mathrm{d} / \mathrm{B}$

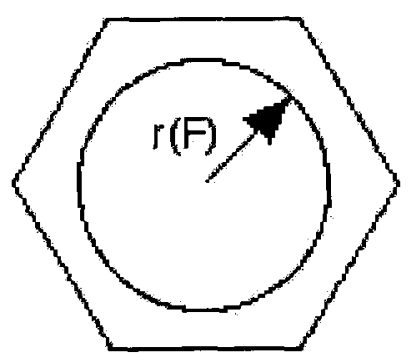

Figure 6. $A_{e f f} / A$ is determined by calculating the area of the circle of radius $r(F)$ relative to the area of the unit cell of dimension $d$ for the square structure, and $d / \sqrt{3}$ for the hexagonal structure. $A_{\text {eff }} / A=1$ when $r=d \sqrt{2} / 2$ in the square case, and when $r=d / \sqrt{3}$ in the hexagonal case.

A plot of these functions is shown in Figure 7.

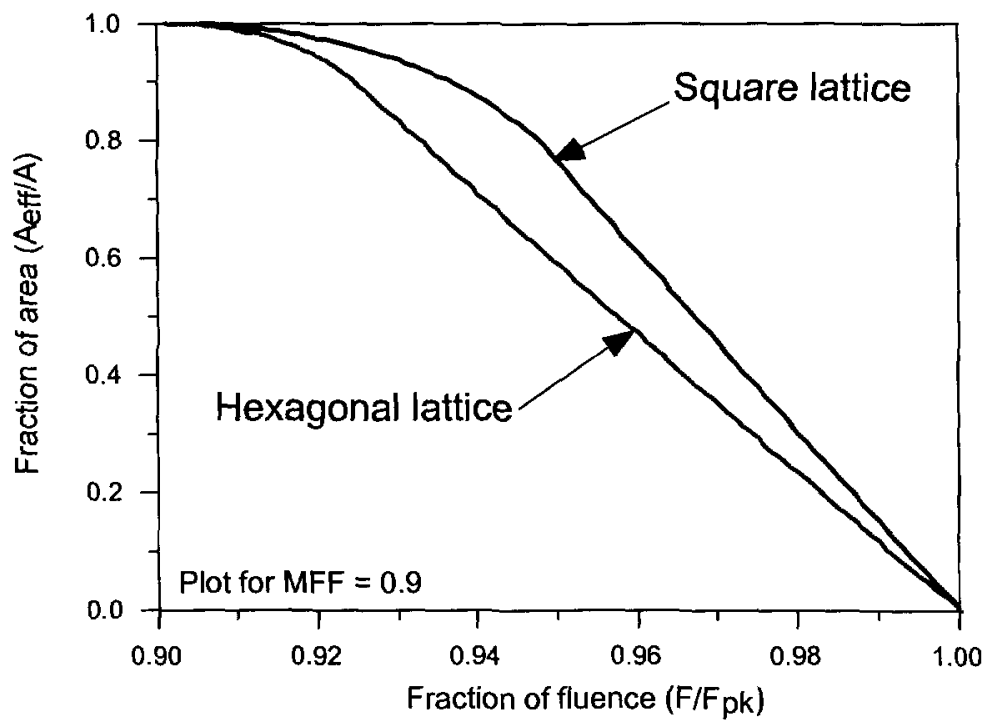

Figure 7. A plot of the fraction of scanned area $\left(A_{\text {eff }} / A\right)$ as a function of fractional peak fluence $\left(F_{p k} / F\right)$. This plot indicates how much area of the sample has been exposed to a fluence of $F / F_{p k}$ or greater. The square lattice covers more effectively than the hexagonal and therefore takes longer to scan.

\section{MULTIPLE PASS SCANNING}

The previous analysis and results assume the sample is scanned using a single pass with a certain overlap. This section addresses the question of whether it is beneficial to use multiple passes with a smaller overlap per pass. Possible advantages include (1) reduced scan time and (2) reduced surface damage due to dragging (see below). The disadvantage of using a smaller overlap, however, is that more scanning fluences may be needed in order to condition properly since the leading edge of the gaussian is being used less effectively. But, for this analysis we can use the results and terminology from the previous section with the addition of a single new variable $p$ for the number of passes:

$\mathrm{p}=$ number of passes (or pass number). 
Thus we still use:
$\phi \quad=1 / \mathrm{e}^{2}$ beam diameter
$\mathrm{A} \quad=$ scanned area
$\mathrm{R} \quad=$ laser repetition rate
$\mathrm{D} \quad=$ distance between adjacent peaks in the scan direction
$f \quad=$ fraction of the peak fluence where two adjacent beams overlap in the scan direction
$\mathrm{N} \quad=$ number of shots per beam diameter in the scan direction
MFF = Minimum Fill Fraction: all of the scanned area is exposed to this fraction of the peak fluence or higher.

To achieve uniform coverage of a sample with either a square or hexagonal pattern, an arbitrary number of passes cannot be applied. It is impossible, for example, to achieve uniform square packing with three passes. To achieve square coverage the number of passes required is:

$$
\mathrm{p}=2^{\mathrm{n}}, \quad \mathrm{n}=0,1,2,3, \ldots \quad \text { (square) }
$$

while for hexagonal coverage it is:

$$
\mathrm{p}=3^{\mathrm{n}}, \quad \mathrm{n}=0,1,2,3, \ldots \quad \text { (hexagonal) }
$$

Figure 8 shows graphically how the square geometry is produced with an increasing number of passes. Note that each additional pass targets areas of minimum fluence coverage (corresponding to the MFF of the previous pass), and that the unit cell dimensions decrease as $\mathrm{d} / \mathrm{Vp}$.
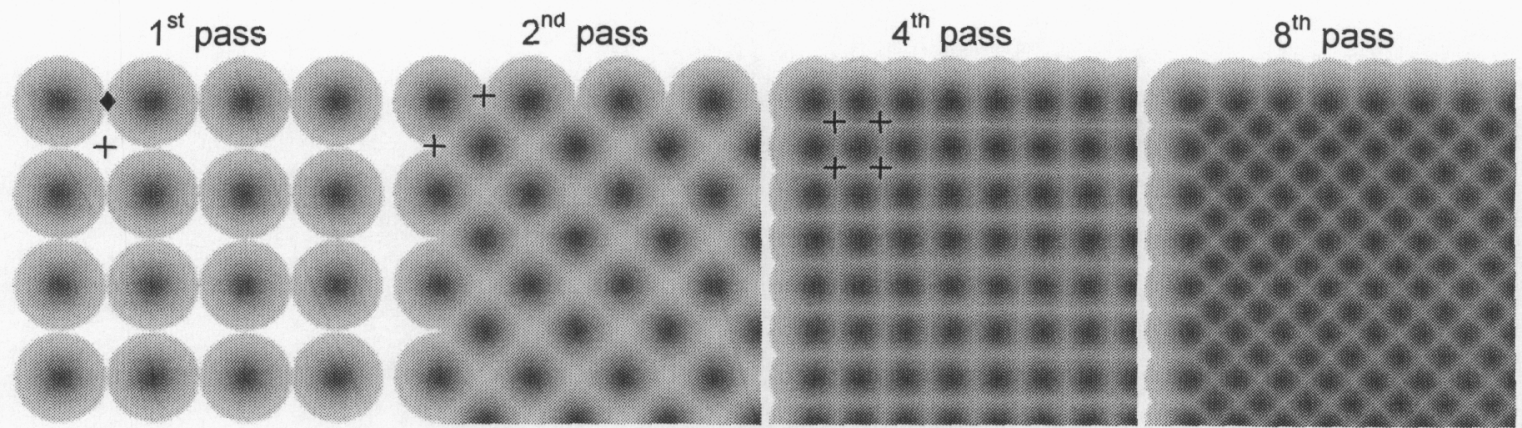

Figure 8. Simulation of Gaussian laser beams in a square pattern after 1,2,4, and 8 passes. Each additional pass is an application of the $1^{\text {st }}$ pass, but shifted with respect to it. The crosses mark the spots where the next image targeted its first beam spot, corresponding to areas of lightest color, i.e. where $\mathrm{F} / \mathrm{F}_{\mathrm{pk}}=\mathrm{MFF}$. The diamond in the first image locates a spot where $\mathrm{F} / \mathrm{F}_{\mathrm{pk}}=f$.

An important piece of information when considering multiple passes is the scan overlap $(f)$ that is required to achieve a desired MFF for a given number of passes. For square coverage, the answer is:

$$
f=(\mathrm{MFF})^{\mathrm{p} / 2} \mathrm{p}=1,2,4,8, \ldots \quad \text { (square) }
$$

while for hexagonal coverage it is:

$$
f=(\mathrm{MFF})^{3 \mathrm{p} / 4} \mathrm{p}=1,3,9,27, \ldots \quad \text { (hexagonal) }
$$


With this we can calculate the total scan time required to scan p passes at scan overlap $f$ to accomplish a given MFF. The total scan time will be the time for one pass multiplied by the number of passes. The single pass scan time is given by $T_{1}=\left(A N^{2}\right) /\left(R \phi^{2}\right)$, where $N$ is given by equation (2). For the square case we can use Eqn. (5) to write

$$
\mathrm{T}_{\text {tot }}=\frac{\mathrm{A} \mathrm{N} \mathrm{N}^{2}}{\mathrm{R} \phi^{2}} \cdot \mathrm{p}=\frac{\mathrm{A}}{\mathrm{R} \phi^{2}} \frac{2 \mathrm{p}}{\ln (1 / \mathrm{f})}=\frac{\mathrm{A}}{\mathrm{R} \phi^{2}} \frac{2 \mathrm{p}}{\ln \left(1 / \mathrm{MFF}^{\mathrm{p} / 2}\right)}=\frac{\mathrm{A}}{\mathrm{R} \phi^{2}} \frac{4}{\ln (1 / \mathrm{MFF})}
$$

and equation (4) is recovered. This analysis can be repeated for the hexagonal case. Since $T_{\text {tot }}$ is independent of $p$, we see that no matter how many passes are taken to achieve a given MFF, the total scan time will be the same for a given packing scheme. Thus factors other than scan time will be more influential in determining how many passes to use to scan an optic, such as scan speed and damage dragging. This is when a surface damage event propagates with successive pulses and is drug along with the beam. Experimentally, it has been observed for DKDP that a scan overlap of $f=0.5$ is sufficient to avoid dragging, while $f=0.9$ is not, indicating that a larger number of passes (and therefore a lower overlap) is desirable. However, the number of passes in a scan will be limited by how fast the stages can move accurately.

\section{THE EFFECT OF POINTING STABILITY AND FLUENCE FLUCTUATIONS}

The two prime factors affecting the ability to cover the scan region uniformly are beam-pointing instability and fluence fluctuations. Both of these effects reduce the value of the minimum fill fraction and lead to more "holes" in the scanned region. The gaussian-beam scanning systems used at LLNL can have worst-case beam-pointing instabilities up to \pm 300 $\mu \mathrm{m}$ and fluence fluctuations up to $\pm 15 \%$. We performed a Monte Carlo analysis to examine the effect of these instabilities on the minimum fill fraction. In the analysis we assumed a normal distribution for both error sources and analyzed 1000 different cell configurations, taking into account cell members and their nearest neighbors, to determine the lowest value of minimum fill fraction. The results of the modeling are given in Table 1 for both square and hex packing patterns and common fill factors of 2 and 4.36 shots/site. Figure 9 shows the effect of beam wander for beam wander and fluence fluctuations up to $\pm 300 \mu \mathrm{m}$ and $\pm 15 \%$ for a square cell with $\mathrm{N}=4.36$.

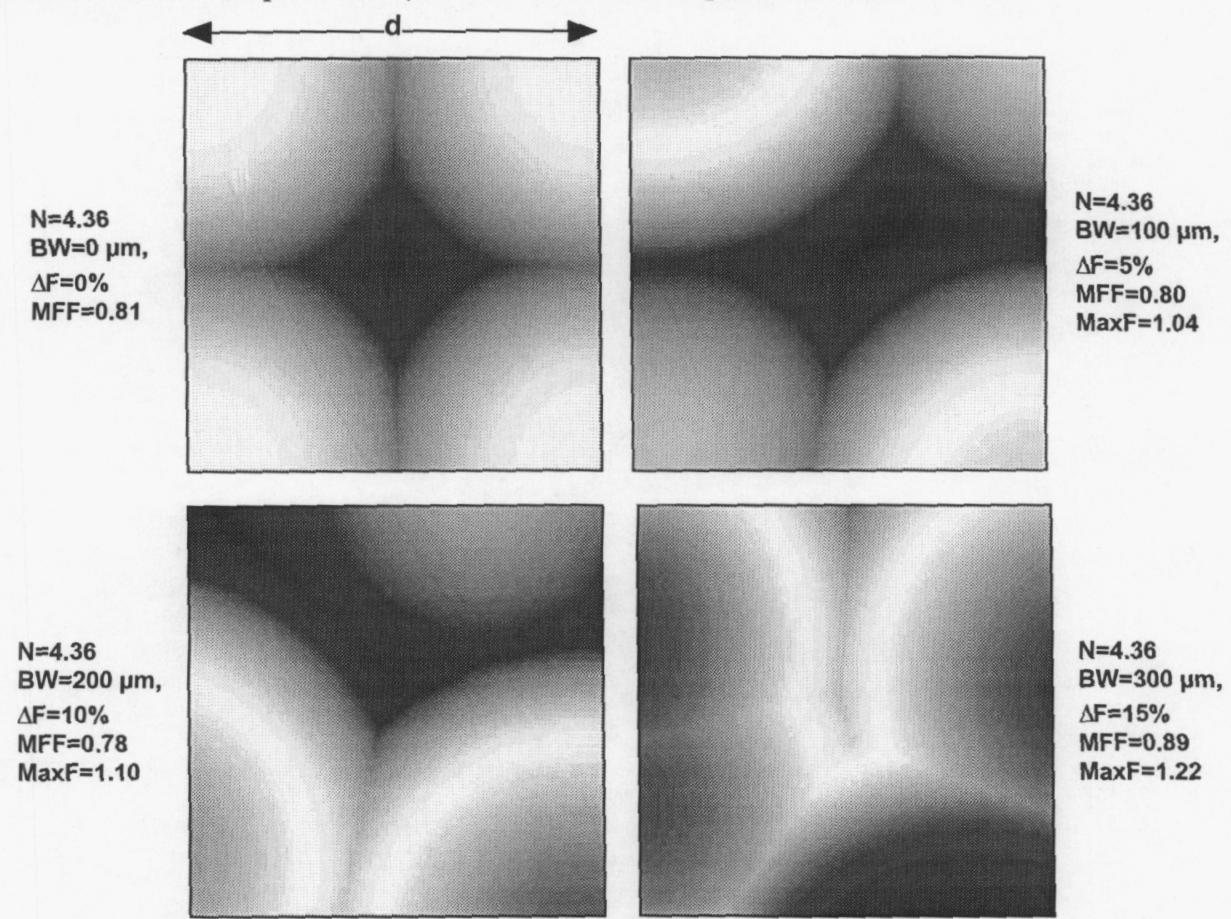

Figure 9. The effect of pointing stability and fluence variations to $300 \mu \mathrm{m}$ and $\pm 15 \%$ on a cell with $\mathrm{N}=4.36$. The color scale of all images covers fluence values of 0.75 to 1.25 . 
The upper left image shows ideally square packed beams for $\mathrm{N}=4.36$ and no beam variations. The beams still occupy the corners of the cell when beam wander is $100 \mu \mathrm{m}$ and fluence fluctuations are $\pm 10 \%$. The four-cornered cell structure is essentially gone when beam wander and fluence variations are greater than $200 \mu \mathrm{m}$ and $\pm 10 \%$.

Inspection of the table reveals that beam wander and fluence instabilities quickly erase any advantage for the hexagonal packing pattern in minimum fill fluence. The table also shows that the fluence holes for the 2 shots/site case are much deeper than for 4.36 shots/site. From LLNL's Phoenix $3 \mathrm{~ns} \mathrm{Nd:YAG} \mathrm{system} \mathrm{scan} \mathrm{parameters} \mathrm{at} 12 \mathrm{~J} / \mathrm{cm}^{2}$ [5], one can expect 117 deep holes of $0.34 \mathrm{~J} / \mathrm{cm}^{2}$ in a NIF optic for the square case and 135 for the hex packing pattern when the fill factor is 2 shots/site. For fill factor of 4.36, there will be 573 shallow holes (square case) and 663 holes (hex) of 5-6 $\mathrm{J} / \mathrm{cm}^{2}$. Practically speaking, with the damage/scanning systems currently available, the experimenter can save some setup time by avoiding hexagonal scans and doing multipass square scans instead.

Table 1. The effect of beam wander and fluence fluctuations on the minimum fill fraction.

\begin{tabular}{|c|c|c|c|c|}
\hline $\begin{array}{c}\text { Fill factor (N) \& } \\
\text { fluence overlap } \\
(f)\end{array}$ & Beam wander $(\mu \mathrm{m})$ & $\begin{array}{c}\text { Fluence fluctuation } \\
(\%)\end{array}$ & $\begin{array}{c}\mathrm{MFF}_{\min } \text { (square)/1000 } \\
\text { cells }\end{array}$ & $\mathrm{MFF}_{\min }(\mathrm{hex}) / 1000 \mathrm{cells}$ \\
\hline $2,0.61$ & 0 & 0 & 0.368 & 0.258 \\
\hline $2,0.61$ & 0 & 15 & 0.247 & 0.515 \\
\hline $2,0.61$ & 100 & 0 & 0.223 & 0.237 \\
\hline $2,0.61$ & 100 & 15 & 0.096 & 0.133 \\
\hline $2,0.61$ & 200 & 0 & 0.049 & 0.062 \\
\hline $2,0.61$ & 200 & 15 & 0.028 & 0.031 \\
\hline $2,0.61$ & 300 & 0 & 0.810 & 0.021 \\
\hline $2,0.61$ & 300 & 15 & 0.528 & 0.869 \\
\hline $4.36,0.9$ & 0 & 0 & 0.728 & 0.589 \\
\hline $4.36,0.9$ & 0 & 15 & 0.591 & 0.738 \\
\hline $4.36,0.9$ & 100 & 0 & 0.638 & 0.472 \\
\hline $4.36,0.9$ & 100 & 15 & 0.523 & 0.526 \\
\hline $4.36,0.9$ & 200 & 0 & 0.549 & 0.487 \\
\hline $4.36,0.9$ & 200 & 15 & 0.493 & 0.487 \\
\hline $4.36,0.9$ & 300 & 0 & & 0.408 \\
\hline $4.36,0.9$ & 300 & 15 & & \\
\hline
\end{tabular}

We also used the simulation to explore the effect of packing density on the distribution of MFF values for the worst-case scenario of $300 \mu \mathrm{m}$ beam wander and $\pm 15 \%$ fluence fluctuation. Figure 10 shows how the cumulative probability distribution for MFF varies as a function of $\mathrm{N}$ and $f$.

The effect of fluence and beam wander can be seen by considering the curve for $\mathrm{N}=2, f=0.61$. Without fluence and wander variations, the expected square-MFF for this is 0.368 . The curve indicates that $71.6 \%$ of the cells will have lower MFF values than ideal while the remaining $28.4 \%$ will have MFF above ideal. In addition, $10 \%$ of the sites will have MFF values lower than 0.15 , so this will yield a scan with many holes. Increasing to $N=4.36, f=0.9$, the ideal square-MFF is 0.81 . The cumulative probability for this value is $69.8 \%$ but $10 \%$ of the sites will have MFF values lower than 0.66 . This curve also shows that $30.2 \%$ for the sites exceed the ideal MFF value. This is due to the combined contributions of complete beam overlap and higher than average fluences. This trend continues as $\mathrm{N}$ is increased. Curiously, the same probability distribution appears for $\mathrm{N}=6.24, f=0.95$ and $\mathrm{N}=8, f=0.97$ where the ideal square-MFF values are 0.90 and 0.94 , respectively. These values correspond to cumulative probabilities of $53.9 \%$ and $72.3 \%$. The $10 \%$ probability for this distribution corresponds to an MFF of 0.805 . The remaining two curves indicate that $\mathrm{N}=10$ will yield MFF $=0.9$ for $98.1 \%$ of the cells, but there is significant probability for MFF values greater than 1 . This is 


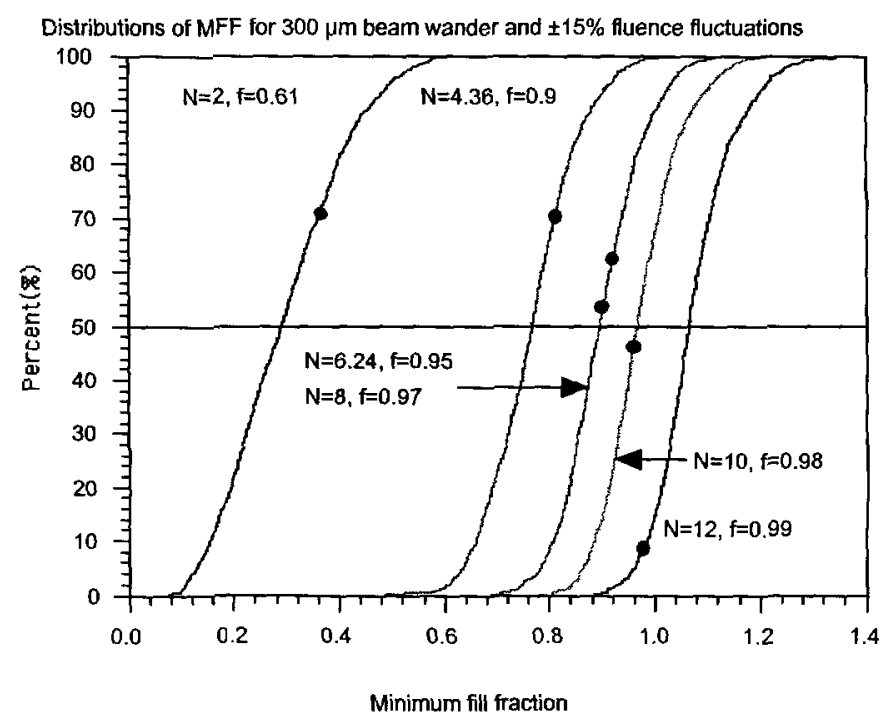

Figure 10. Cumulative probability distributions for MFF with beam fluence and beam wander variations of $\pm 15 \%$ and $300 \mu \mathrm{m}$ respectively. The black dots indicate the ideal MFF for the fill factor.

significant because these values are due mainly to fluence fluctuations and there is a danger that the sample could experience damage. In addition, there is a large time penalty associated with these laser variations. A NIF scan with the ideal $\mathrm{MFF}_{\text {ideal }}=0.9(\mathrm{~N}=6.24, f=0.95)$ using the 3 ns Phoenix laser would require 6.55 hours. It requires $10^{2} / 6.24^{2}=2.6$ times as long (or 17.6 hours) to scan at $\mathrm{N}=10$ to achieve the desired $\mathrm{MFF}=0.9$, and over $50 \%$ of the sites would be scanned at higher than ideal MFF. Of course, this represents what we view as a worst-scenario for beam wander and fluence fluctuations. More refined numerical simulations can be done to model actual laser systems. This will require detailed statistical studies of beam wander and fluence fluctuations, not only to determine the magnitudes of these effects, but also the governing probability distributions (which may not be gaussian).

\section{SUMMARY}

We have developed the nomenclature to describe the raster scanning process using gaussian beams in terms of number of shots/beam diameter (N) and the minimum fill fraction (MFF) for square and hexagonal packing schemes. The main conclusions of the work are that there is an inherent trade-off between degree of fluence uniformity and scan time. If the scan system did not possess any fluctuations, the rectangular gaussian beam offers lower scan times compared to the square or hexagonal packing schemes for round gaussian beams. For round gaussians the hexagonal packing would be $23 \%$ faster than square for a given MFF value, but with beam wander and shot-to-shot fluence fluctuations typical of our scanning systems, this advantage is negated. From a practical standpoint it is easier to execute a multipass square series of scans and live with the (shallow) fluence holes created by fluctuations.

The key factor in determining the number of passes to achieve a desired MFF is whether damage is dragged along with the scan. For KDP, we have seen that beam overlap factors of 0.5 do not cause significant dragging while it is more prone to $f=0.9$. Also central to this question is whether a high fill factor achieves better conditioning due to the higher number of shots on the leading edge of the beam. If this were the case, then slow scanning using excimers, with appropriately shaped beams, would be preferable. This issue will be addressed by experiments presented in reference 5 .

\section{ACKNOWLEDGEMENTS}

Work performed under the auspices of the U.S. Department of Energy by Lawrence Livermore National Laboratory under Contract No. W-7405-ENG-48. 


\section{REFERENCES}

1. Christopher J. Stolz, Lynn M. Sheehan, Stephen M. Maricle, Sheldon Schwartz, "A study of laser conditioning methods of hafnia silica multilayer mirrors," in Laser-Induced Damage in Optical Materials: 1998, Gregory J. Exarhos, Arthur H. Guenther, Mark R. Kozlowski, Keith Lewis, and M. J. Soileau, Editors, SPIE Vol. 3578, 144153 (1999).

2. Raymond M. Brusasco, Bernie M. Penetrante, John E. Peterson, Stephen M. Maricle, Joseph A. Menapace, "UV laser conditioning for the reduction of 351-nm damage initiation in fused silica," in Laser-Induced Damage in Optical Materials: 2001, Gregory J. Exarhos, Arthur H. Guenther, Keith Lewis, M. J. Soileau, and Christopher J. Stolz, Editors, SPIE Vol. 4679, 48-55 (2002).

3. Michael J. Runkel, Kurt Neeb, Michael Staggs, Jerome M. Auerbach, Alan K. Burnham, "The results of raster-scan laser conditioning studies on DKDP triplers using Nd:YAG and excimer lasers," in Laser-Induced Damage in Optical Materials: 2001, Gregory J. Exarhos, Arthur H. Guenther, Keith Lewis, M. J. Soileau, and Christopher J. Stolz, Editors, SPIE Vol. 4679, 368-383 (2002).

4. Michael J. Runkel, James J. De Yoreo, Walter D. Sell, David Milam, "Laser conditioning study of KDP on the Optical Sciences Laser using large area beams," in Laser-Induced Damage in Optical Materials: 1997, Gregory J. Exarhos, Arthur H. Guenther, Mark R. Kozlowski, and M. J. Soileau, Editors, SPIE Vol. 3244, 51-63 (1998).

5. Unpublished results on post-test inspection of prototype NIF Final Optics Assembly tested on the Beamlet laser.

6. Sheldon Schwartz, Michael D. Feit, Mark R. Kozlowski, Ron Mouser, "Current $3 \omega$ Large Optic test procedures and data analysis for the quality assurance of National Ignition Facility optics," in Laser-Induced Damage in Optical Materials: 1998, Gregory J. Exarhos, Arthur H. Guenther, Mark R. Kozlowski, Keith Lewis, and M. J. Soileau, Editors, SPIE Vol. 3578, 314-321 (1999).

7. Michael J. Runkel, Justin R. Bruere, Walter D. Sell, Timothy L. Weiland, Douglas E. Hahn, Mike C. Nostrand, "Effects of pulse duration on bulk laser damage in 350-nm raster-scanned DKDP," to be published in Laser-Induced Damage in Optical Materials: 2002, Gregory J. Exarhos, Arthur H. Guenther, Keith Lewis, M. J. Soileau, and Christopher J. Stolz, Editors, SPIE Vol. 4679, (2003). 